\title{
Additive Manufactured Bioplastics for Conceptual Models of Knee Customized Prostheses
}

\author{
DELIA ALEXANDRA PRISECARU*, DANIEL BESNEA, EDGAR MORARU1', SORIN CANANAU \\ Politehnica University of Bucharest, 313 Splaiul Independentei, 060042 Bucharest, Romania
}

The article presents a promising technology for medical applications - additive technology by thermoplastic extrusion. Some conceptual models for knee prostheses of different constructional forms have been made using FDM technology. Different materials, including biocompatible, were considered and different $3 D$ printing equipment was used to choose the variant with the most optimal characteristics. These conceptual models can have various beneficial applications in medicine, improving the quality and functionality of personalized prostheses.

Keywords: additive manufacturing, bioplastics, conceptual models, knee prostheses

In the medical field, Rapid Prototyping processes are used for diagnostic or didactic purposes, to obtain a physical model of the studied object (anatomical replica) or to reduce the time of prosthesis production. One of the perspective applications is the additive manufacturing technology in the field of personalized medical implantology. Possible applications of 3D printing technologies in medicine are as follows: tissue engineering, bone regeneration, organ printing, tissue biofabrication; medical research and bioengineering; controlled medication: controlled release of drugs. Another good example would be planning a difficult operation based on a 3D model, before the patient enters the operating room. The ability to obtain a physical replica of a particular anatomical structure is also an advantage in the planning and simulation of difficult operations, such as those in the field of craniomaxillofacial surgery, where it works with complex and variable geometries. Although virtual models can be useful in planning a very complex intervention or in its three-dimensional simulation, the use of a physical object is even more efficient. The physical model is useful to study much of the risks and difficulties that may arise in the case of real intervention, to view the movements allowed during the operation or to evaluate the area in which it will intervene. In addition, the model can serve as a master or negative in the implementation of implants. The aforementioned advantages are added to numerous applications of rapid fabrication procedures in the manufacture of medical instruments, implants and prostheses, operations planning, tissue engineering or dentistry. Also, the RP medical models offer tactile and visual information, especially useful in the communication between doctors or between doctor and patient.

In the case of knee prostheses, the problem of adapting it according to the patient's particularities is very important. A geometric nonconformity, a choice of a prosthesis with materials that are not the most suitable to be accepted by the body, but also the problem of functioning in time, with the prolongation of the life of the prosthesis in optimum conditions until its replacement, represents a challenge of researchers in the field to provide an optimal solution and comfort of life for each patient who will be the subject of such arthroplasty.

The issue of materials is particularly important in this context. As shown in Fig. 1 (a), the real model of the joint is very complex. The technical solution, to get closer to the real model of this human joint, a highly complex one, located between the femur and tibia, includes an association of materials for the components of the joint.
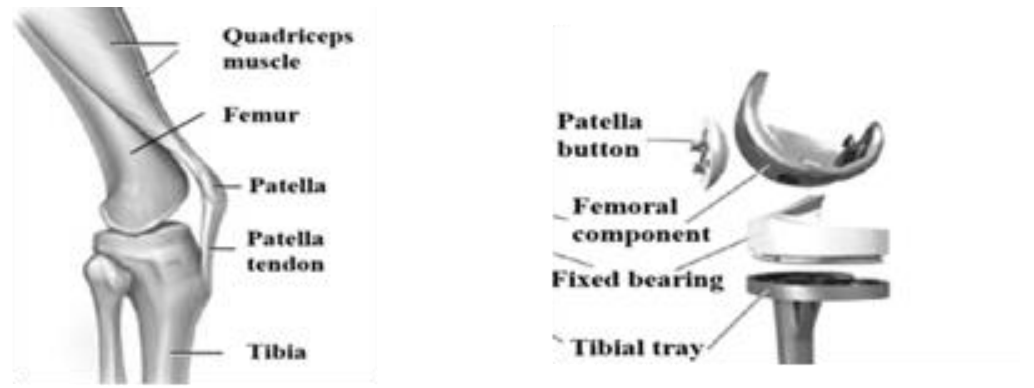

Fig.1 (a)Knee joint anatomy, (b)Knee prosthesis (arthoplasty)

(adapted Junfen Shi (2007)) - Finite Element Analysis of total knee replacement considering the gait cycle load and malalignement, $\mathrm{PhD}$ Thesis University of Wolverhampton, 2007, https://core.ac.uk/download/pdf/1931943.pdf , pag.6(fig.1(a)), pag 31(fig.1(b))

\footnotetext{
*email: delia.prisecaru@upb.ro
} 
As illustrated in Fig. 1(b), the real model is replaced by a technical solution comprising three components: the femoral component, the tibial tray and the bearing interface (fixed bearing) which is integral with the tibial insert. The femoral component is typically made of Cobalt-Chrome alloy which is a very hard and durable material. This metal is also able to be highly polished at the surface, being one of the few metals that is allowed to be used inside the human body; group that includes Stainless Steel, Titanium-Aluminium alloy, and pure Platinum or Titanium. This quality improves the tribological behaviour of the joint. The tibial tray is usually made of Titanium or also Cobalt-Chrome. There are arguments for choosing each of these materials. The tibial bearing component is the component between the femor and tibial one and has a major part in bearing all the weight of the human body, under functioning conditions. The bearing is made of a plastic material usually ultra high molecular weight polyethylene (UHMWPE). Moreover, tibial components are often made of plastic material. This type of plyethylene has some key features that are extremely valuable in the present case, such as: low friction factor, excellent chemical resistance, springy toughness and, very important for the making process, a versatile processing. There are several designs to fix the plastic bearing component to the tibial tray. The current research deals with the design and use of plastic material for the knee prosthesis. The research is also based on the use of Rapid Prototyping technique [1-9].

\section{The 3D modeling software solution}

The 3D design of the custom implant was realized with the help of the SolidWorks software. SolidWorks uses the method of generating solid bodies through features, one of the most used 3D body modeling techniques. SolidWorks is also a viable solution for the design of different components and assemblies, being a mechanically parameterized $3 \mathrm{D}$ design program, highly performing at present.

Three conceptual models of personalized prostheses have been designed, all having as a common element a fixed median part, a fixing part and a mobile upper part that can be moved and controlled on the median surface. The model shown in Fig. 1, (a), (b) illustrates a fixed bearing design in the femoral component through a cone-like geometry. The model in Fig. 1, (c), (d), a frequently used one, shows a fixed bearing component that is made of a metal support and an upper part of the plastic material, inserted in the support. The models in Fig. 1, (e), (f) represents the proposed ones, with some of the components made by means of the RP technique.

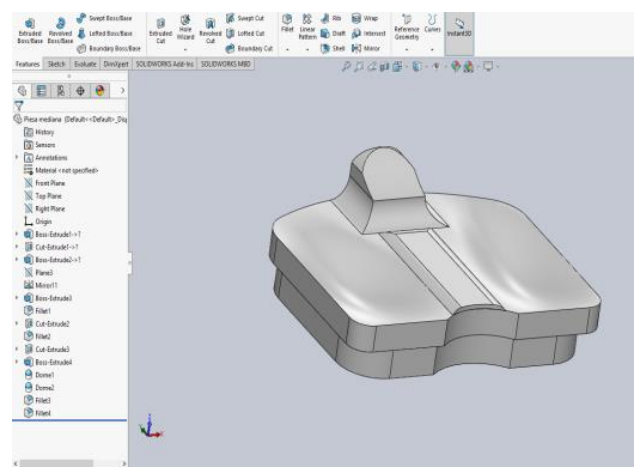

(a)

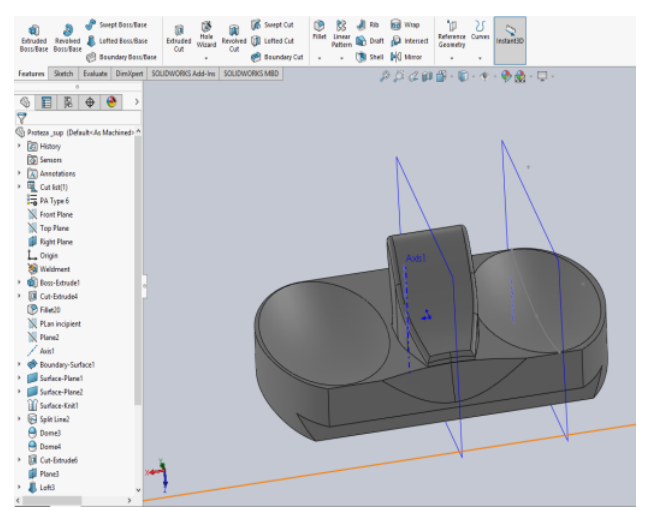

(c)

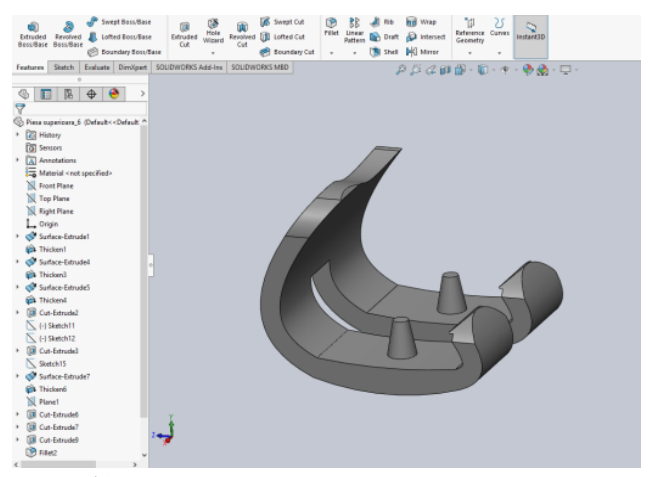

(b)

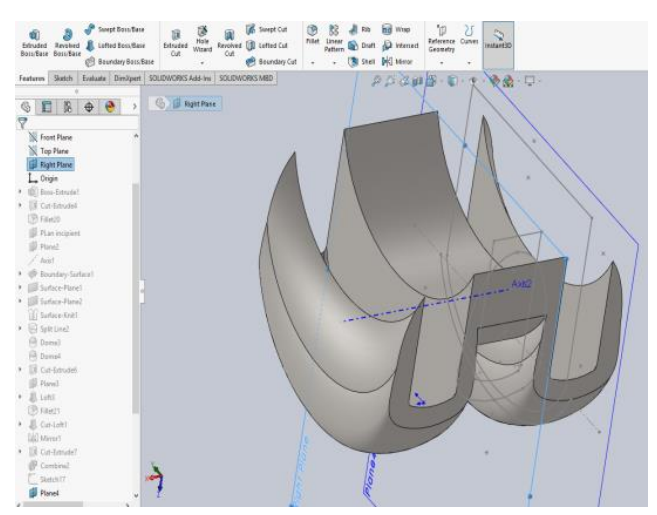

(d) 


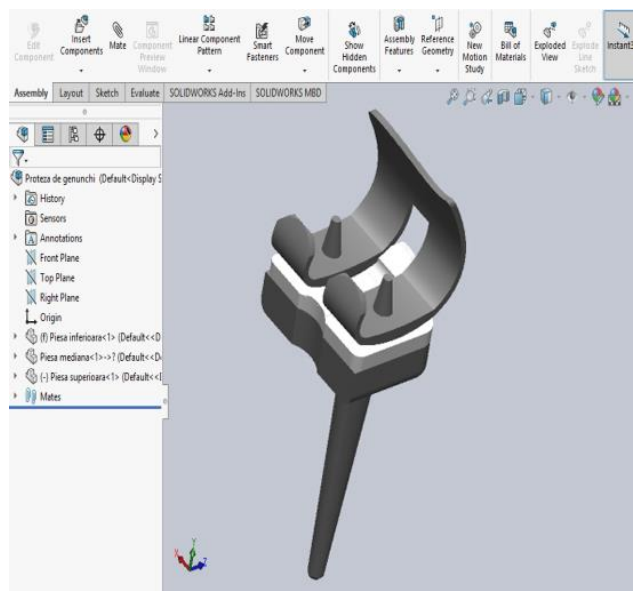

(e)

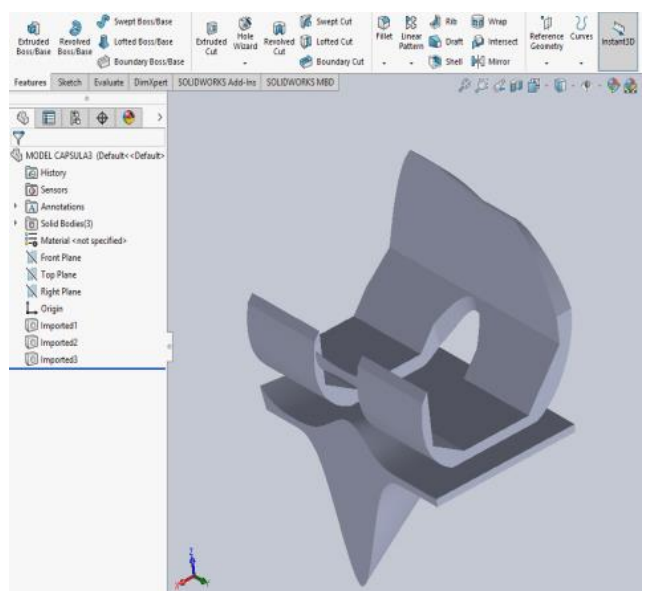

(f)

Fig. 2. 3D modeling using the SolidWorks software of the three types of customized knee prostheses

\section{Realization of the customized knee prostheses by additive technologies}

Thermoplastic Extrusion (FDM-Fused Deposition Modeling) is an additive manufacturing process that belongs to the material extrusion family. The processes based on the extrusion of materials uses a wire made of different materials, which heats on a temperature of several degrees below melting temperature, after which it reduces its diameter to $0.12-0.15 \mathrm{~mm}$ by extruding it in a device deposition. The device moves in the XOY plane to materialize a section of the 3D virtual model. In FDM technology, an object is constructed by selectively depositing molten material in a predetermined layer-by-layer form.

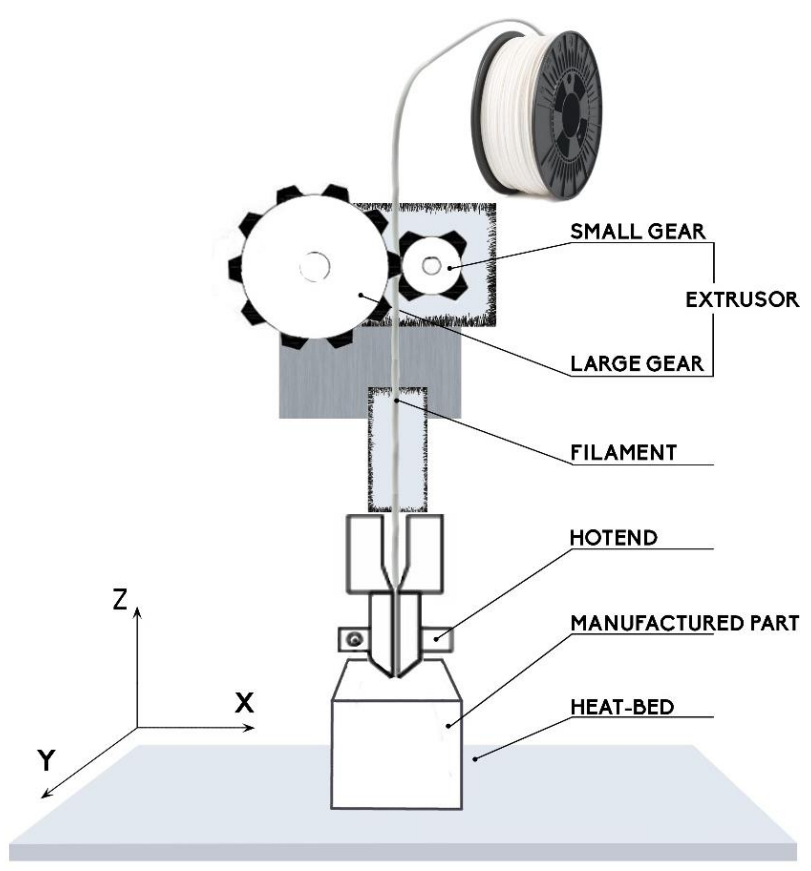

Fig.3 FDM principle of functioning made after Rodriguez's example [10]

The materials used for the printing of custom knee prostheses are PLA (Easyprint) thermoplastic polymers, ABS and a biocompatible material (Smartfil medical), in the form of a $1.75 \mathrm{~mm}$ diameter filament. PLA material (polylactic acid) Easyprint is a biopolymer, a biodegradable material made of renewable raw materials, being able to produce parts with detailed surfaces with minimal deformations. The technical characteristics of the material are as follows: printing temperature: $180-215^{\circ} \mathrm{C}$, platform temperature: 0-60 $\mathrm{C}$. ABS (acrylonitrile-butadiene-styrene) is an oilbased plastic with a higher surface hardness and impact-resistant. ABS is recyclable, but it remains a non-sustainable option due to its petroleum-based origin, but presenting an excellent stiffness and excellent dimensional stability, at a low production cost. For 3D printing of knee prosthesis models, the impact-resistant Golden material was used: printing temperature: $220 \pm 25^{\circ} \mathrm{C}$, platform temperature: $65-80^{\circ} \mathrm{C}$. Smartfil medical is a high-quality material specially designed for medical applications. This filament has USP certification class VI or ISO 10993-1, a filament 
biocompatible with the human body. This class allows material contact with the human body for a certain period of time. This high quality material allows the production of parts with a high degree of detail and without deformations. Technical characteristics of the material: printing temperature: $240 \pm 10^{\circ} \mathrm{C}$, platform temperature: $80-100{ }^{\circ} \mathrm{C}$, printing speed: $40-50 \mathrm{~mm} / \mathrm{s}$.

For the physical realization of custom knee prosthesis models, several 3D printers were used:

- MakerBot Replicator (Fig.4a) with the main technical characteristics: layer resolution: 270 microns; positioning accuracy: on XY: 11 microns, on Z: 2.5 microns; filament diameter: 1,75mm; extrusion nozzle diameter: $0.4 \mathrm{~mm}$;

- Wanhao 4S Duplicator (Fig.4b) with the main technical characteristics: print size (mm) $225 \times 145 \times 150$; diameter $0.4 \mathrm{~mm}$; extruders 2; layer thickness $01-0.5 \mathrm{~mm}$; print speed $40 \mathrm{~mm} / \mathrm{s}$; print temperature $\max 260^{\circ} \mathrm{C}$; filament diameter $1.75 \mathrm{~mm}$;

- Delta 3D Printer: Up to 50 microns layer resolution; maximum print speed: $150 \mathrm{~mm} / \mathrm{s}$. maximum size of printed object: 180 (diameter) x 300 (height) $\mathrm{mm}$; metal extruder E3D V5 J-head; filament material: PLA (recommended); $1.75 \mathrm{~mm}$ diameter filament; XYZ positioning accuracy: $0.01 \mathrm{~mm}$; nozzle diameter: $0.4 \mathrm{~mm}$; recommended extruder temperature: $200-240^{\circ}$ Celsius;

The realization of several pieces on the printers used in this study has outlined a list of advantages and disadvantages as illustrated in Table 1.

Table 1

ADVANTAGES AND DISADVANTAGES OF USED EQUIPMENT

\begin{tabular}{|c|c|c|}
\hline \multirow{2}{*}{ Wanhao Duplicator 4S } & Advantages & Disadvantages \\
\hline & - Wide range of materials & - Low precision and quality \\
& - Heated bed & - High noise level \\
& - Accessible price & \\
\hline Makerbot & - Precision and quality & - High price of the equipment \\
& - Easy to remove sacrificial layers & - Only PLan be printed \\
& - Distance control & \\
\hline Delta & - Ergonomic design & - Small work area \\
& - Large dimensions on Z axis & - Difficult calibration \\
\hline
\end{tabular}

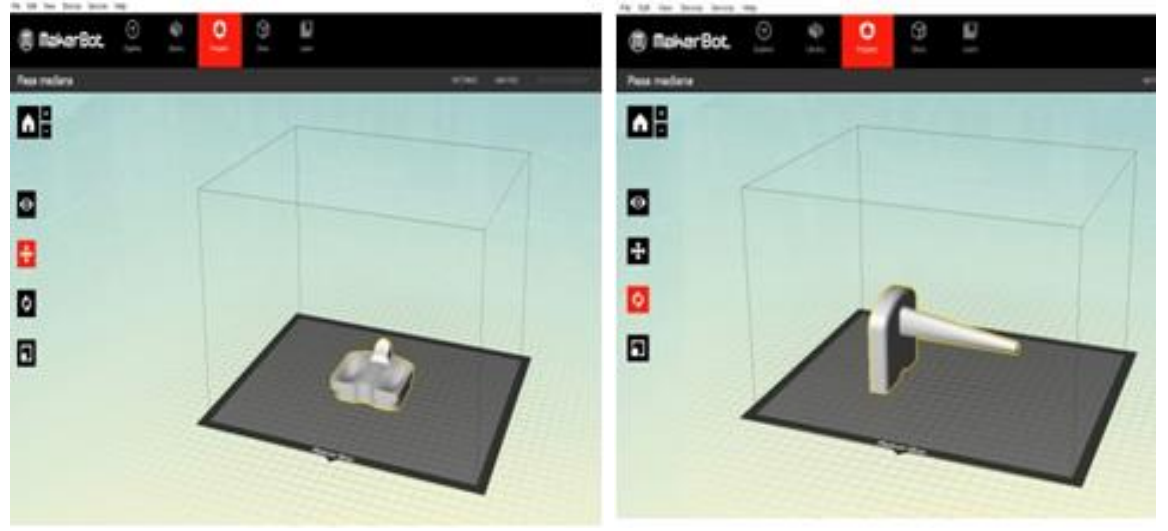

a. MakerBot interface 

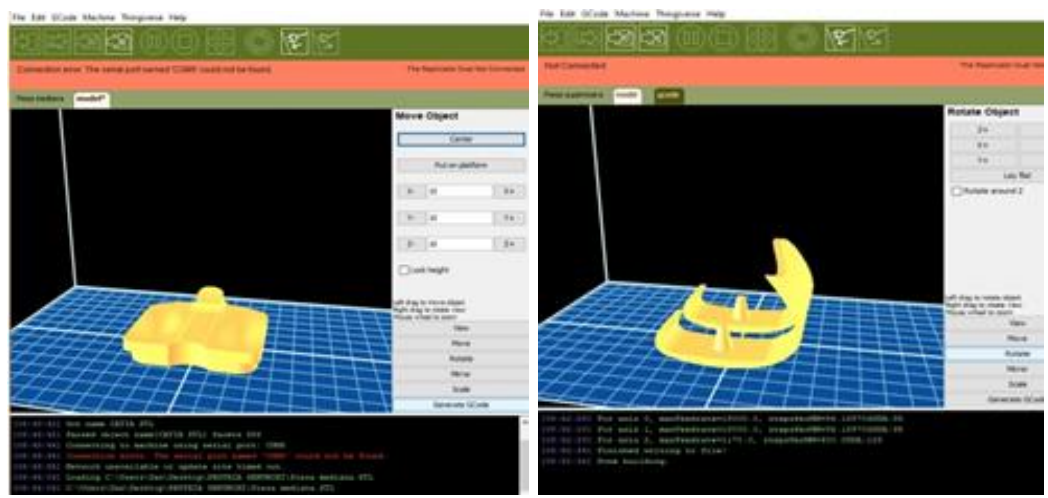

b. RepetierHost interface

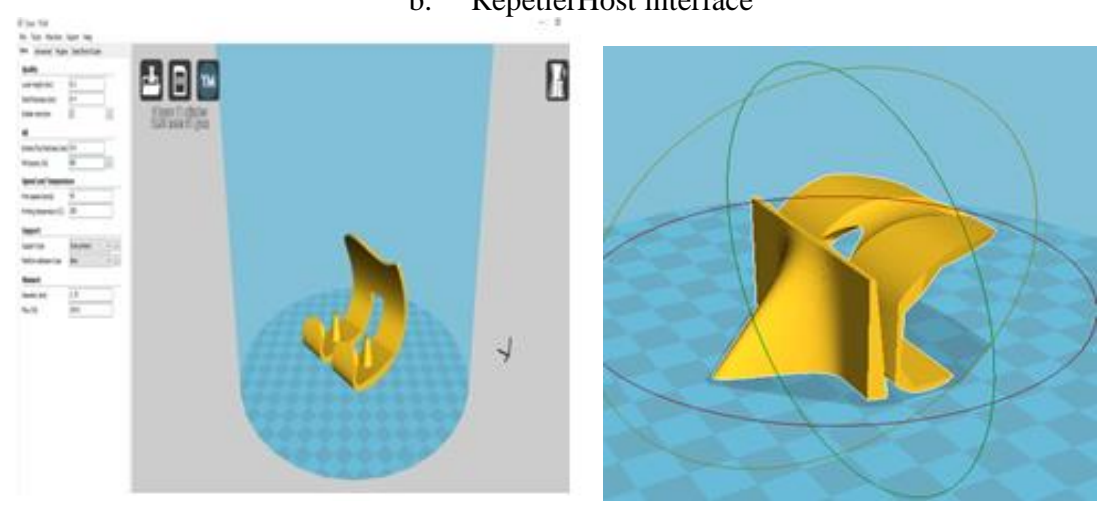

c. Cura interface

Fig. 4 Positioning the knee prosthesis models saved in .stl format in the printing space of the three types of printers: a- MakerBot interface for the MakerBot Replicat or printer;

b - Repetier Host interface for Wanhao Duplicator 4 S printer; c- Cura interface for Delta printer

The Wanhao Duplicator 4S 3D printer allows printing complex objects, using support structures where needed or creating objects in two colors or two different materials (ABS, PLA etc.), with two extruders and two possibilities of heating the work platform at $120^{\circ} \mathrm{C}$. The 3D MakerBot printer offers professional printing quality, offers a high print speed up to $150 \mathrm{~mm} / \mathrm{s}$ provided by a precise and safe mechanism. The Delta type control system is the second most used system in the 3D printer industry. 3D printers in the Delta system also use the Cartesian coordinate system, yet modified. The movement of the arms of such a printer is done at a fixed, predetermined angle, and this greatly increases the printing speed. Also, the printing surface is fixed, it cannot be moved, which is useful for certain parts. In addition to the much improved print speed, the 3D printer in the Delta system also has the great print height on the vertical axis as an important advantage.

\section{Effort simulation of the customized knee prosthesis conceptual model}

For the verification and comparison regarding the mechanical behavior of the proposed custom model, with a reference model, the model in Fig.2 (c), (d) was chosen as reference.

The contact between bodies involves information concerning kinematic conditions and dynamic conditions. Mechanical contact and supported load conditions is a problem which involves the materials in contact, because it depends on the elastic properties of the bodies in contact and their geometry. To verify the models under load, first, the model presented in Fig.2(c),(d) is analysed.
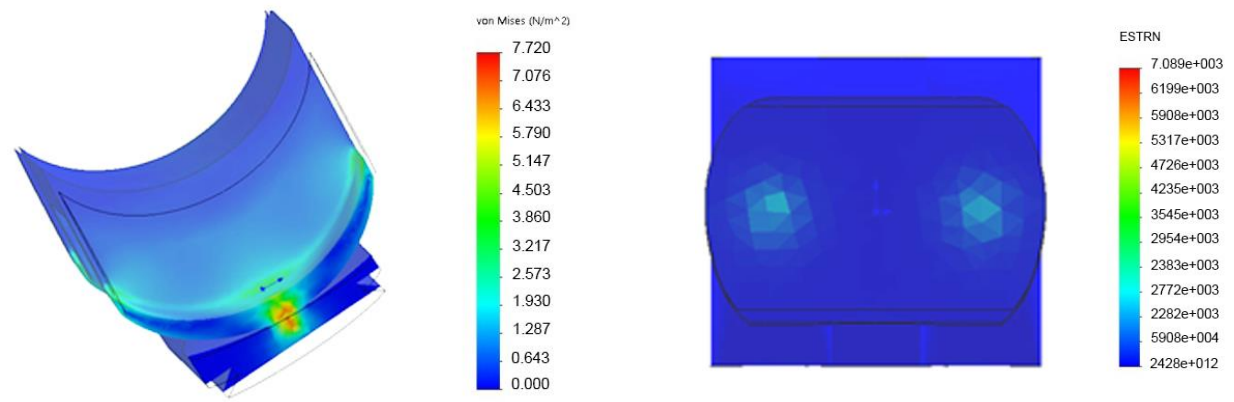

Fig. 5 (a) simulation of contact stress, (b) simulation of contact strain 
In Fig. 5(a) we can observe two contact situations: one with insertion of polyethylene (UHMWPE) tibia component and the second contact between the femoral component and the polyethylene insert (UHMWPE). Also, in Fig. 5(b) we can observe the fixed surfaces (geometry) represented by the tibial component and the down side of the plateau.

The idea of the new proposed model is to create a prosthesis which can reduce a part of the stress due to contact by createing a new design of the parts. As shown in Fig. 6(a), the new model, due to a greater elasticity and to the RP techinque to create the femoral component, presents a vulnerability in the region of bone meshing. Still, the contact with the fixed bearing is realized in better conditions. This is also explained by the improved area of the contact surface, as seen in Fig. 6(b)
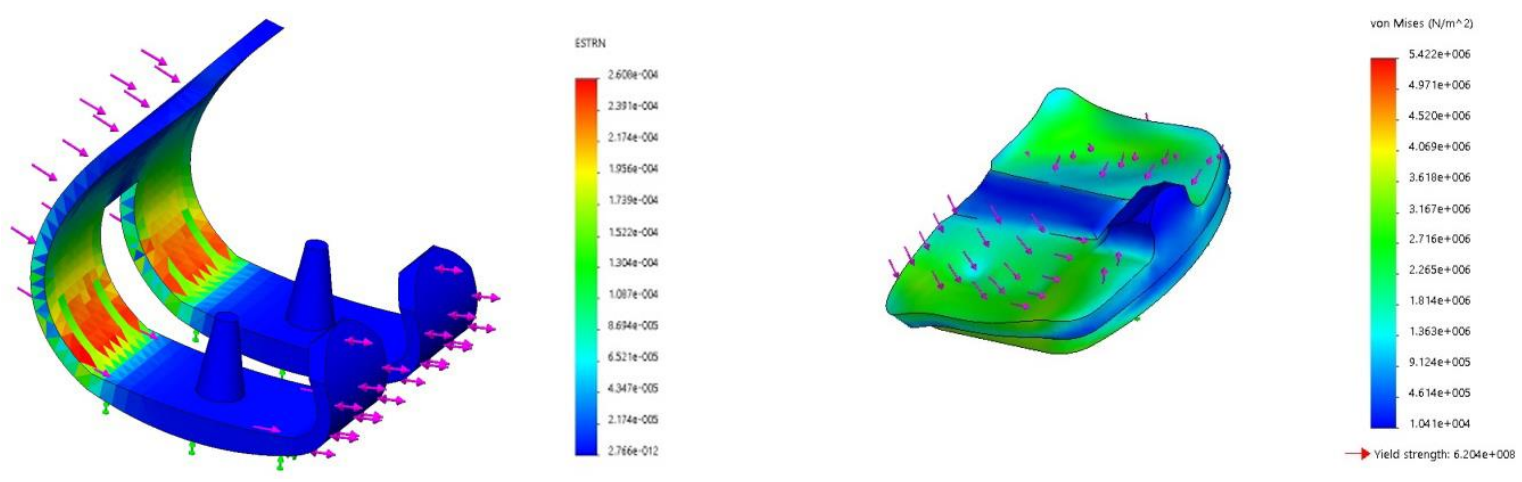

Fig. 6. Simulation of the knee prosthesis

Figure 7 represents the conceptual models for the knee prostheses made by additive technologies.

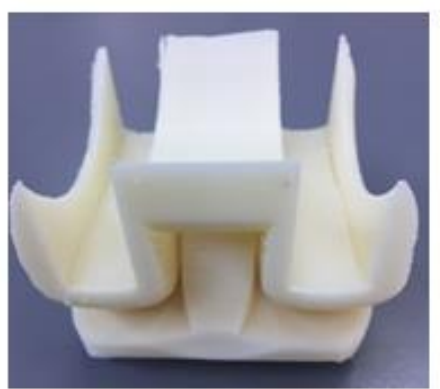

a

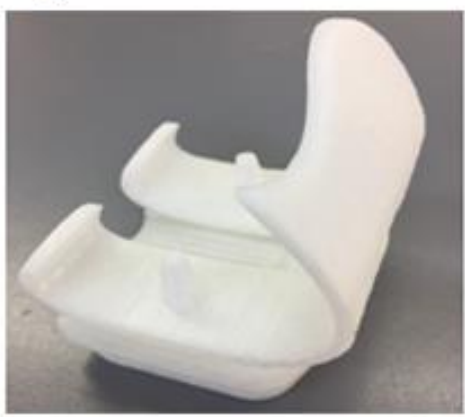

d

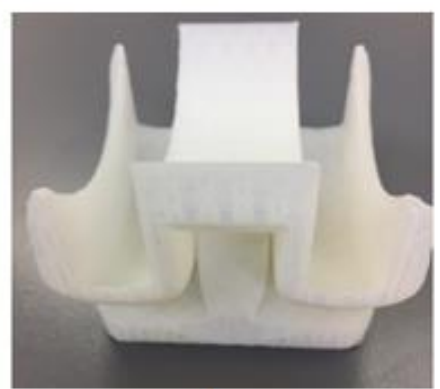

b

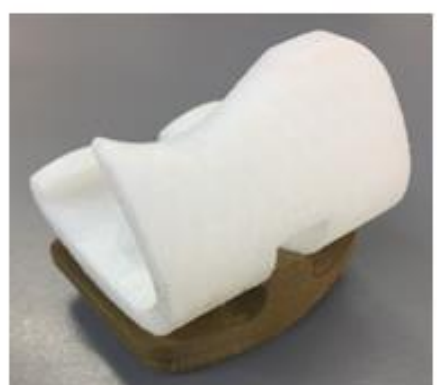

C

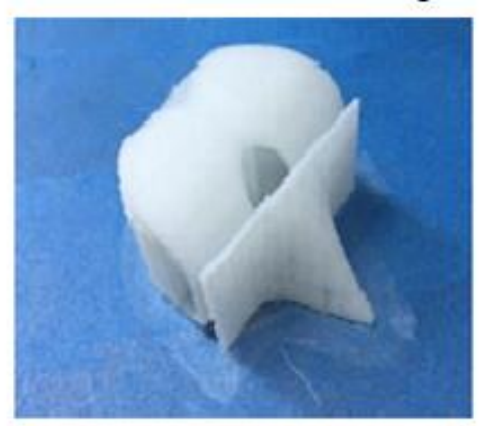

e

Fig.7. 3D conceptual models of knee prostheses obtained : a) Material Smartfil medical median and superior prosthesis, demonstrative model; 3D printer Wanhao Duplicator 4S; FDM technology; b) Material PLA median and superior prosthesis, demonstrative model; Delta 3D printer; FDM technology; c) Material ABS median prosthesis and PLA superior prosthesis, demonstrative model ; Delta 3D printer; FDM technology; d) Material PLA median and superior prosthesis, demonstrative model; MakerBot 3D printer; FDM technology; e) Material PLA median and superior prosthesis, demonstrative model;

Delta 3D printer; FDM technology 


\section{Conclusions}

Smartfil medical is a biomaterial that allows the development of personalized medical models that can come into contact with the human body for a short period of time, representing a provisional medical solution for serious cases that require complicated surgery. Rapid Prototypind technologies represent a solution for the rapid realization of customized medical prostheses from usual ABS and PLA materials that allow obtaining conceptual models, demonstrating low cost prices that can further validate a design solution in the field of personalized implantology. Using a thermoplastic material with adequate mechanical properties, these can become a low-cost solution for the prosthesis of patients with a moderate level of activity. The development of customized prostheses through additive technologies is limited to the range of materials that can be used and not to its design. However, given the scientific progress in the field of technology and materials science, surely these manufacturing methods will have a hard say in the orthopedic and prosthetic field. Future studies will aim to make custom prostheses by means of other additive methods. Also, raw materials in other forms (liquid, powder) will be considered.

\section{References}

1.BESNEA, D., DINU, E., MORARU, E., SPANU, A., RIZESCU, C., CONSTANTIN, V., Experimental Researches Regarding the Manufacturing of New Thermoplastic Materials Used in Additive Technologies, Mat. Plast., 56, no.1, 2019, p.167-170.

2.BERCE P., BALC N., CAIZAR C., PACURAR R., RADU A.S., BRATEAN S., FODOREAN I., Tehnologii de fabricatie prin adaugare de material si aplicatiile lor, Editura ACADEMIEI ROMANE, Bucuresti, 2014.

3.POPA, A., FAUR, N., HLUSCU, M., BELIN, C., Evaluation of the Mechanical Properties of the Samples Made by FDM 3D Printing, Mat. Plast., 56, no.3, 2019, p.500-504

4.SZUHANEK, C, MIHAI, A.M., SARBU, A., PRICOP, M., 3D Printed Surgical Guides Used in Orthodontics, Mat. Plast., 56, no.3, 2019, p.657-659

5.FABIAN, Z., KADAR, K., PATONAY, L., NAGY, K., Application of 3D Printed Biocompatible Plastic Surgical Template for the Reconstruction of a Nasoalveolar Cleft with Preoperative Volume Analysis, Mat. Plast., 56, no.2, 2019, pp.413-415

6.MUHLFAY, G., FABIAN, Z., NEAGOE, R., URSUlA, HORVATH K., Applications of 3D Planning, Plastic Materials and Additive Manufacturing in Functional Rehabilitations in the Head and Neck Surgery, Mat. Plast., 55, no.3, 2018, p.431-433

7.MICLAUS, R., REPANOVICI,A., ROMAN, N., Biomaterials: Polylactic Acid and 3D Printing Processes for Orthosis and Prosthesis, Mat. Plast., 54, no.1, 2017, pp.98-102

8.PAHONIE, R., STEFAN, A., ADOCHIEI, I.R., COSTUlEANU, C.L., ANDRUSEAC, G.G., UNGUREANU, G., SARDARU, D.P., Experimental Characterisation of the Mechanical Properties of Lightweight 3D Printed Polymer Materials for Biomechanical Application in Ankle-Foot Orthosis, Mat. Plast., 54, no.2, 2017, p.396-401

9.NAGIB, R., SZUHANEK, C., MOLDOVEANU, B., NEGRUTIU, M.L., SINESCU, C., BRAD, S., Custom Designed Orthodontic Attachment Manufactured Using a Biocompatible 3D Printing Material, Mat. Plast., 54, no.4, 2017, p.757-758

10.RODRIGUEZ J.P., CORTES C.A., RODRIGUES E., Atlas_GcodeSender: a Graphical User Interface for Control of a Robotics Additive Manufacturing Platform, 2018 IEEE International Conference on Mechatronics and Automation (ICMA).

Manuscript received: 11.11 .2019 